\title{
Metal-dielectric-metal plasmonic resonators for active beam steering in the infrared
}

\author{
Enes Battal ${ }^{1, *}$ and Ali Kemal Okyay ${ }^{1,2}$ \\ ${ }^{1}$ Department of Electrical and Electronics Engineering, Bilkent University, Ankara 06800, Turkey \\ ${ }^{2}$ UNAM-Institute of Materials Science and Nanotechnology, Bilkent University, Ankara 06800, Turkey \\ *Corresponding author: battal@ee.bilkent.edu.tr
}

Received November 19, 2012; accepted January 31, 2013;

posted February 19, 2013 (Doc. ID 180158); published March 14, 2013

\begin{abstract}
Active beam-steering devices near the optical frequencies have long been sought after due to their applications in communication, defense, and display technologies; however, the challenge lies in achieving actively tunable structures near these frequencies. An array of metal-dielectric-metal plasmonic resonators is demonstrated as a dynamic beam-steering device to operate at midinfrared wavelengths. We numerically demonstrate continuous-angle beam steering of $8.75^{\circ}$ by making use of tunable properties of silicon as the active dielectric. The proposed device achieves a refractive index insensitive divergence angle and it operates in a $650 \mathrm{~nm}$ wide spectral window around $10 \mu \mathrm{m}$ wavelength. The results of this Letter pave the way to exploiting active beam steering in various applications at midinfrared wavelengths. (c) 2013 Optical Society of America

OCIS codes: $110.1080,230.6046,230.4555,240.6680,310.6628$.
\end{abstract}

In the past, an enormous amount of research effort has been spent on steering light beams. Active control of the propagation direction of light is at the very heart of holographic image-generation technologies [1] and novel optical communication schemes [2]. For these purposes, researchers have investigated reconfigurable diffraction gratings [3], movable microlens arrays [4], tunable waveguides [5] , electro-optic prisms []ㅡ, and leaky wave antennas [7]. Active control of refractive index is the most commonly employed method to obtain nonmechanical beam steering. Magneto-optic []], nonlinear [9] phase change [10] materials, and doped semiconductors [11] and liquid crystals [3] have been used to actively control the refractive index in resonant structures. Plasmon resonators offer mode profiles in highly spatially confined volumes and considerably increase the field-matter interaction. Recently, innovative beam-steering devices exploiting strong resonance properties of surface plasmons (SPs) have been demonstrated at near- [12] and mid- [13] infrared wavelengths utilizing subwavelength metallic slits coupled with liquid crystals and thermally reconfigurable semiconductors.

Metal-dielectric-metal (MDM) structures have been shown to exhibit strong plasmon resonance at a broad range of wavelengths [14]. SPs can be guided at metaldielectric interfaces of MDM structures and enable very strong localization of light inside very small volumes. The resonant characteristics of these structures can be modified by varying the refractive index of the dielectric [14]. Therefore, MDM resonators can be used as actively controlled phase shifters for beam-steering devices. Yet, these structures are not exploited to realize active beam-steering devices.

In this Letter, we propose a beam-steering device based on phased-array-like MDM structures operating at midinfrared wavelengths. We theoretically explore the beam-steering characteristics of the proposed device through analyzing the effects of changing dielectric properties on its radiation pattern. The device is shown to achieve continuous-angle beam steering with divergence angle insensitive to refractive index. In addition, the beam-steering performance of the proposed device is relatively invariant within a narrow band around a chosen wavelength. The structure can be fabricated using optical lithography, thin film deposition, and etching techniques.

We investigate an array nanoantenna structures formed by MDM resonators shown in Fig. 1. We simulate five array elements for the sake of computational simplicity. Each element consists of a sandwiched dielectric slab between a top and a bottom metal slab. The slabs are assumed semi-infinite extending in the $z$ direction. As a proof-of-concept demonstration, we set dielectric $\left(t_{d}\right)$ and metal $\left(t_{m}\right)$ thicknesses to 300 and $100 \mathrm{~nm}$, respectively. The center-to-center distance $(d)$ between each element of the array is kept constant at $2300 \mathrm{~nm}$. We perform our analysis by the two-dimensional finitedifference time-domain (FDTD) method using FDTD Solutions from Lumerical Inc. The light is incident from the top with transverse-magnetic polarization; i.e., the electric-field vector is along the $x$ axis, and its wavelength is assumed to be $\lambda=10 \mu \mathrm{m}$. A total-field scattered-field source is used in order to illuminate a finite structure with a plane-wave-like light so that each element is excited in phase. Perfectly matched layers

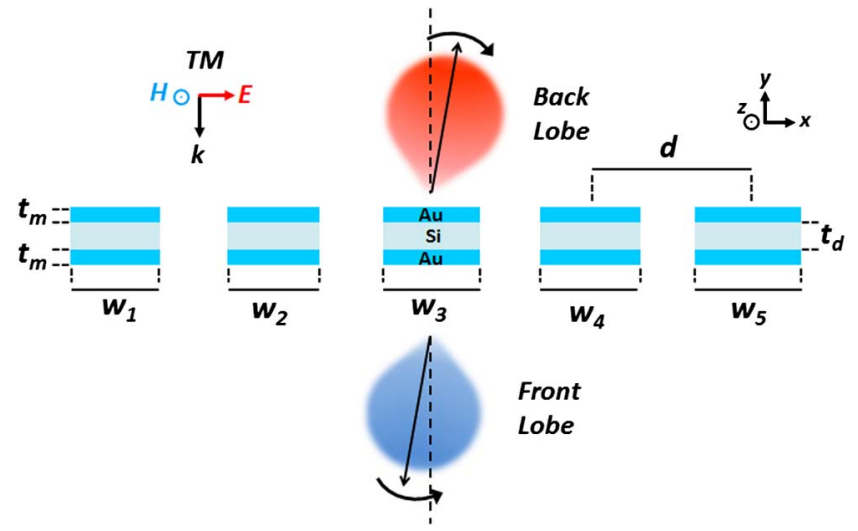

Fig. 1. (Color online) Array of MDM resonators with different widths for active beam steering. 
are used as the boundary conditions for all dimensions. The structure is covered with a mesh region of $5 \times 5 \mathrm{~nm}$ unit-mesh size. Far-field radiation patterns are extracted by projecting the scattered radiation in near field to far field at $1 \mathrm{~m}$ away from the structure. The directivity and beam-steering angle are calculated with respect to lobe centers. The top and bottom metals are chosen to be gold $(\mathrm{Au})$, and the surrounding medium is assumed to be air. Due to the low-cost process, silicon $(\mathrm{Si})$ is assumed as the active dielectric. The optical constants of $\mathrm{Au}$ and Si from Palik [15] are used in the simulations. When the electron concentration of $\mathrm{Si}$ is increased from intrinsic levels up to the order of $10^{18} \mathrm{~cm}^{-3}$, its refractive index $\left(n_{\mathrm{Si}}\right)$ decreases from 3.42 to 3.27 and its extinction coefficient $\left(k_{\mathrm{Si}}\right)$ is always below 0.032 [15], which is neglected in this work (i.e., we take $\overline{k_{\mathrm{Si}}}=0$ ). This approximation slightly overestimates the radiated intensity since the losses are neglected. These carrier concentration levels are preferred because of their feasibility in the standard CMOS process [16]. The carrier concentration of Si can be controlled by thermal [17], electrical [11], or optical [18] means. Regardless of the control method, this Letter focuses on the beam-steering characteristics of the proposed device.

Finite-width MDM structures are plasmonic waveguides that can support coupled surface plasmon Fabry-Perot (SP-FP) modes at the top and bottom metal dielectric interfaces [shown in Fig. 2(a)] when the right-propagating SPs (R-SPs) and the left-propagating SPs (L-SP) interfere constructively [14]. The resonant interference condition of SPs is given by

$$
k_{0} n_{\mathrm{eff}} w=m \pi+\varphi,
$$

where $k_{0}$ is the wave vector of the incident light, $n_{\mathrm{eff}}$ is the effective refractive index inside the waveguide, $w$ is the width of the slab, $m$ is an integer, and $\varphi$ is the phase picked up by SPs due to reflection at the ends of the waveguide. For intrinsic $\mathrm{Si}\left(n_{\mathrm{Si}}=3.42\right), n_{\mathrm{eff}}$ at the wavelength of $\lambda=10 \mu \mathrm{m}$ is calculated to be 3.72 from its dispersion relation [14].

First, we investigate the radiation characteristics of a single array element, depicted in Fig. 2(a). We set the width of the slab, $w$, to $1320 \mathrm{~nm}$ in order to satisfy the

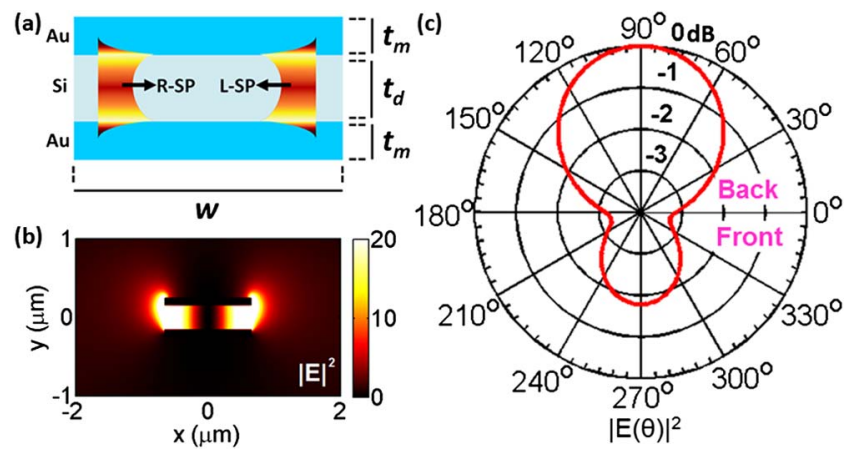

Fig. 2. (Color online) (a) Single MDM resonator element supports right- (R-SP) and left- (L-SP) propagating SPs. (b) Electricfield intensity profile shows a standing wave pattern due to Fabry-Perot resonance of SPs. (c) Dipole-like radiation pattern of the single MDM resonator. interference condition (for $\lambda=10 \mu \mathrm{m}$ ) given in Eq. (1) with a first-order resonance $(m=1)$. The E-field intensity distribution of the structure depicted in Fig. 2(b) shows a very strong confinement inside the waveguide due to SP-FP mode excitation as expected. The radiation pattern of the structure [Fig. 2(c)] resembles that of a dipole with a slight asymmetry. The directivity of the front lobe is $0.053 \mathrm{dBi}$, whereas it is $1.83 \mathrm{dBi}$ for the back lobe. Half-power beam widths (HPBWs) of the front and back lobes are both higher than $150^{\circ}$. Both lobes are symmetric with respect to their center and centered about the $y$ axis due to the lack of asymmetry in the structure around the $y$ axis.

In phased-array microwave antennas, beam steering is achieved by controlling phase shifts between consecutive elements of the array. In the MDM structure, the phase shift introduced by each element is controlled by varying the refractive index of the dielectric layer. The elements are chosen with increasing widths in order to introduce different phase shift. We take $w_{1}, w_{2}, w_{3}, w_{4}$, and $w_{5}$ to be $1320,1380,1440,1500$, and $1560 \mathrm{~nm}$, respectively. These width values are determined by a parameter sweep around the resonant value (1320 nm). Compared to the single MDM structure at $n_{\mathrm{Si}}=3.42$, the front and back lobes of the array MDM structure are deflected by $-4.7^{\circ}$ and $-8.3^{\circ}$, respectively, due to different phase shift contributions from each element. The asymmetry in the radiation intensity between the front and back lobes is preserved; however, the radiation intensity of the front lobe has decreased due to the off-resonance elements of the array. Directivity is increased to $3.96 \mathrm{dBi}$ for the front lobe and to $7.11 \mathrm{dBi}$ for the back lobe. In addition, HPBW is greatly decreased to $42^{\circ}$ and $42.6^{\circ}$ for the front and back lobes, respectively. Such improvements in the directivity and HPBW are attributed to the array factor.

We achieve active beam steering by varying the refractive index of the dielectric layer of the array MDM structure. An angular steering of $8.75^{\circ}$ from $265.25^{\circ}$ to $274^{\circ}$ for the front lobe is obtained when $n_{\mathrm{Si}}$ is changed from 3.42 to 3.27, as can be seen in Fig. 3(a). The characteristic is a continuous angular steering as shown in Fig. 3(b). In the case of the back lobe, the steering characteristics are relatively poor $\left(2.35^{\circ}\right)$. The maximum beam-steering range corresponding to unit change in the refractive index, $\Delta \theta_{\max } / \Delta n$, is an important factor for evaluating
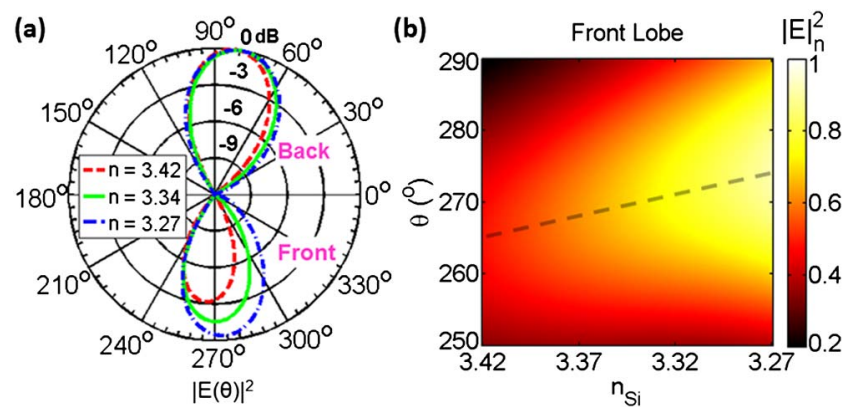

Fig. 3. (Color online) (a) Front lobe of the phased-array-like MDM structure shifts by $8.75^{\circ}$ and the back lobe shifts by $2.35^{\circ}$ when $n_{\mathrm{Si}}$ changes by 0.15 . (b) Normalized radiation intensity $\left(|E|_{n}^{2}\right)$ at the center of the front lobe shifts continuously and its amplitude increases by decreasing $n_{\mathrm{Si}}$. 

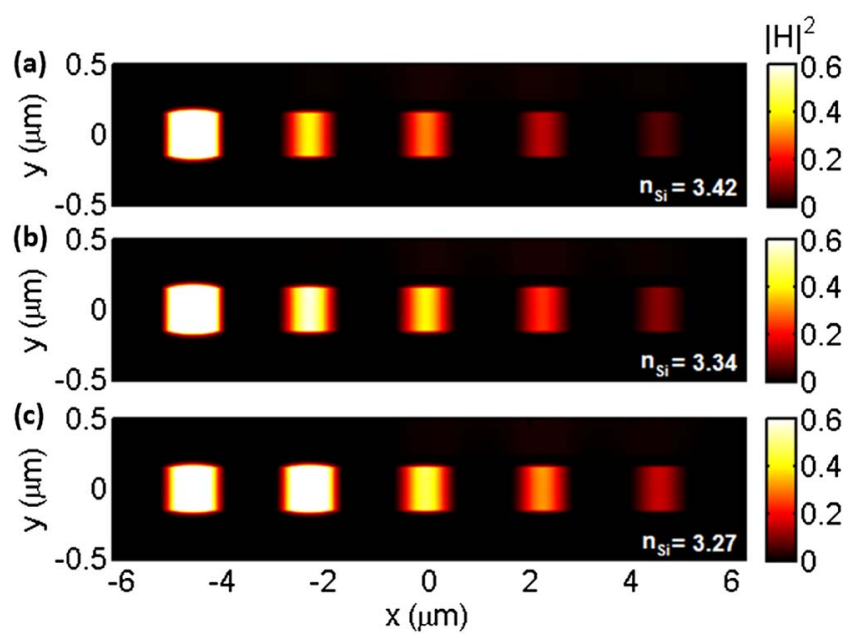

Fig. 4. (Color online) Magnetic-field intensity $\left(|H|^{2}\right)$ profile indicates a shift of resonant behavior toward wider elements (to right) as $n_{\mathrm{Si}}$ decreases intermittently from (a) 3.42 to (b) 3.34 and (c) 3.27 .

beam-steering performance. Here, we report a maximum value of $\Delta \theta_{\max } / \Delta n=58.3^{\circ}$ for the front lobe.

The resulting phased-array-antenna-like behavior can also be deduced from the change in the localization of the incident light between the array elements. This behavior is apparent in the $H$-field intensity profiles extracted for $n=3.42,3.34$, and 3.27 in Figs. $\underline{4(\mathrm{a})}-\underline{4(\mathrm{c})}$, respectively. The observed active beam steering is arising from SP-FP resonance shifts toward wider elements (higher $w$ ) as required from Eq. (1) when the refractive index of the dielectric is decreased.

The HPBW of the front lobe is relatively immune to refractive index changes only with the increment of $3.4^{\circ}$, and directivity increases up to $5.05 \mathrm{dBi}$ with decreasing $n_{\mathrm{Si}}$. The boost in directivity is attributed to higher power radiation in the front lobe due to stronger overall resonance at lower refractive indices (i.e., improved matching between the incident and the transmitted media) as seen in Fig. 4. Directivity can be further increased by adding more array elements to the MDM structure, which is beyond the scope of this work.

Assuming the same refractive index range for $\mathrm{Si}$, we show that the beam-steering range $\left(\Delta \theta_{\max }\right)$ of the front lobe of the proposed array MDM structure is relatively immune to spectral changes with a full width halfmaximum (FWHM) of $650 \mathrm{~nm}$ in the $9.68-10.33 \mu \mathrm{m}$ wavelength range as depicted in Fig. $\underline{5}$. This spectrum can be useful for coupling beam-steering devices with commercial $\mathrm{CO}_{2}$ lasers [19].

In this Letter, we numerically demonstrated an active beam steering based on an array of MDM plasmonic resonators operating at midinfrared wavelengths. The proposed structure exhibits continuous-angle beam steering by $8.75^{\circ}$ with a 0.15 change in the refractive index, while the divergence angle of the beam is preserved. Steering is achieved for a $650 \mathrm{~nm}$ wide spectral window around $10 \mu \mathrm{m}$ wavelength. The device concept proposed here can be realized using common microfabrication techniques; however, the potential impact of process variability on device performance is not investigated.

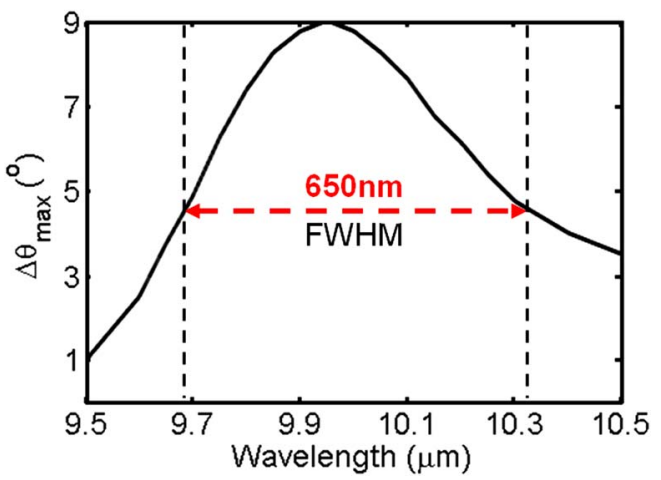

Fig. 5. (Color online) Maximum beam-steering angle, $\Delta \theta_{\max }$, is relatively preserved within a FWHM of $650 \mathrm{~nm}$ around the operation wavelength $(\lambda=10 \mu \mathrm{m})$.

Moreover, as resonant MDM structures can be achieved at a wide range of frequency bands (visible, terahertz, gigahertz), active beam steering at such frequency bands can be achieved. Therefore, this work points to a fruitful room for realization of active beam-steering devices.

This work was supported in part by the European Union Framework Program 7 Marie Curie IRG Grant 239444, COST NanoTP, and TUBITAK Grants 109E044, 112M004, and 112E052. The authors acknowledge support from TUBITAK-BIDEB.

\section{References}

1. N. S. Holliman, N. A. Dodgson, G. E. Favalora, and L. Pockett, IEEE Trans. Broadcast. 57, 362 (2011).

2. R. Keil, M. Heinrich, F. Dreisow, T. Pertsch, A. Tunnermann, S. Nolte, D. N. Christodoulides, and A. Szameit, Sci. Rep. 1, 94 (2011).

3. H.-C. Jau, T.-H. Lin, R.-X. Fung, S.-Y. Huang, J. H. Liu, and A. Y. G. Fuh, Opt. Express 18, 17498 (2010).

4. M. Holz and W. C. Goltsos, Opt. Eng. 29, 1392 (1990).

5. M. Jarrahi, R. F. W. Pease, D. A. B. Miller, and T. H. Lee, Appl. Phys. Lett. 92, 014106 (2008).

6. J. F. Lotspeich, IEEE Spectrum 5, 45 (1968).

7. Q. Song, S. Campione, O. Boyraz, and F. Capolino, Opt. Express 19, 8735 (2011).

8. V. V. Temnov, Nat. Photonics 6, 728 (2012).

9. A. E. Attard, Appl. Opt. 38, 3239 (1999).

10. K. Appavoo and R. F. Haglund, Nano Lett. 11, 1025 (2011).

11. C. Qiu, X. Ye, R. Soref, L. Yang, and Q. Xu, Opt. Lett. 37, 3942 (2012).

12. D. de Ceglia, M. A. Vincenti, and M. Scalora, Opt. Lett. 37, 271 (2012).

13. D. C. Adams, S. Thongrattanasiri, T. Ribaudo, V. A. Podolskiy, and D. Wasserman, Appl. Phys. Lett. 96, 201112 (2010).

14. S. I. Bozhevolnyi and T. Stndergaard, Opt. Express 15, 10869 (2007).

15. E. D. Palik, Handbook of Optical Constants of Solids (Academic, 1997), Vol. 3, pp. 174-179.

16. International Technology Roadmap for Semiconductors, http://www.itrs.net.

17. G. Cocorullo, M. Iodice, and I. Rendina, Opt. Lett. 19, 420 (1994).

18. Q. Xu and M. Lipson, Opt. Express 15, 924 (2007).

19. Access Laser, http://www.accesslaserco.com/pdf/CO2 Spectrum and Tunable Lasers.pdf. 